\section{Arzneimittel und Wege zu einer gesunden und schönen Haut}

D ie meisten Lehrbücher der Dermatologie gehen von den einzelnen Krankheitsbildern aus und erörtern die möglichen Therapieverfahren in diesem Rahmen. Das vorliegende Werk geht einen ganz anderen Weg. Untergliedert in topische und systemische Dermatika

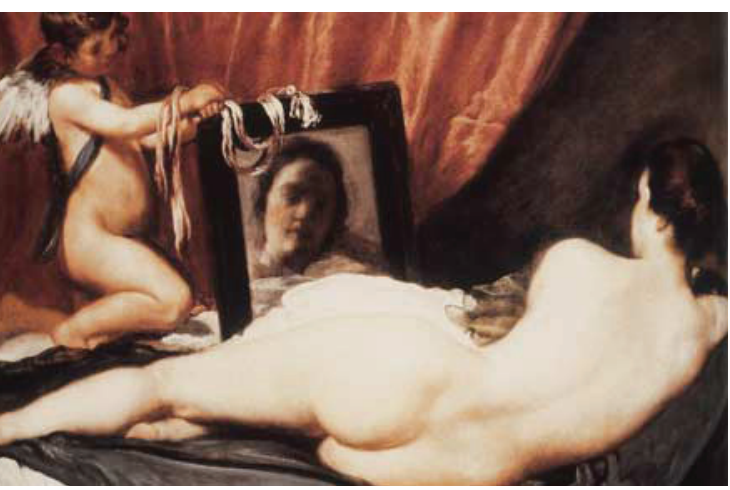

Nur in einer gesunden Haut fühlt man (frau) sich wohl.

sowie Kosmetika werden die einzelnen Wirkstoffgruppen in Form von Monographien von namhaften Experten besprochen. Eine strenge Gliederung ermöglicht bei 76 (!) Autoren dennoch einen einheitlichen Rahmen. So ist jede dieser Monographien unterteilt in Chemie, Analytik, Pharmakokinetik und dynamik, Toxikologie, Dosis und Art der Applikation, Präparate, unerwünschte Wirkungen, Auswahlkriterien und Alternativen, Einsatzhäufigkeit sowie Wertung aus persönlicher Sicht des Experten. Diese Form der Darstellung erleichtert es dem Leser, sich rasch einen profunden Überblick über den jeweiligen Wirkstoff zu verschaffen. Das ausführliche und aktuelle Literaturverzeichnis trägt ein Übriges dazu bei, weitergehendes Interesse zu befriedigen. Die Autoren bieten nicht nur theoretische Informationen zur Wirkweise von Substanzen, sie zitieren auch die jeweiligen Studien, die bei einer Substanz die Wirksamkeit belegen. So ist dieses Werk auch hinsichtlich des Anspruchs der Evidenz-basierten Medizin auf dem aktuellsten Stand. Alles in allem wird deutlich, welch reichhaltiges therapeuti- sches Arsenal dem Dermatologen heute zur Verfügung steht. Das Buch spannt einen Bogen von schon seit langem bekannten Therapeutika wie Dithranol und Zinkverbindungen zu modernen und teilweise noch experimentellen Therapieverfahren wie der Gabe von Zytokinen, Thalidomid oder Immunglobulinen.

Im Kapitel Kosmetika kann sich der Leser nicht nur über Hautreinigungsmittel und Deodorantien informieren, auch die Wirkungsweise von Selbstbräunern und Lichtschutzmitteln wird erklärt. In der heutigen Zeit sind die Patienten durch Verbraucherinformationen aus der Presse zunehmend besser aufgeklärt, deshalb ist es für den Dermatologen unabdingbar, über wissenschaftlich fundierte Informationen über Kosmetika zu verfügen, um kompetent Stellung beziehen zu können.

In einem allgemeinen Kapitel werden unter anderem die Definitionen von Dermatika und Kosmetika, Grundzüge der Magistralrezeptur sowie die Anwendung in Schwangerschaft und Stillzeit

W elcher praktisch tätige Arzt, sei es ein Internist oder Allergologe, hat nicht Patienten vor sich, die über einen allgemeinen Juckreiz und eine trockene Haut (Xerodermie oder Xerosis cutis) klagen? Die Lehrbücher der Dermatologie geben für die Diagnostik und Therapie dieser scheinbar eher belanglosen Beschwerden kaum Hilfe.

Umso wertvoller ist dieses 100 Seiten starke kleine Buch. Es stellt die medizinischen Grundlagen der so genannten trockenen, empfindlichen Haut oder Mischhaut sowie die praktikablen, nicht invasiven Methoden zur Bestimmung der Hauttrockenheit dar. Die Dermatosen, die mit einer trockenen Haut einhergehen, umfassen das atopische Ekzem, die Ichthyosen, die Pomadenkruste, Palmoplantarkeratosen, Medikamenten-induzierte Verhornungsstörungen, Kopfschuppen und schließ- abgehandelt. Ganz besonders zur Abrundung des Werkes tragen die Ausführungen zur Nutzen-Risiko-Bewertung und Nutzen-Aufwand-Bewertung von Dermatika und Kosmetika bei. In Zeiten eines zunehmenden Kostendrucks im Gesundheitswesen kann die Bedeutung der Pharmako-Ökonomie nicht hoch genug eingeschätzt werden.

Den Herausgebern ist es hervorragend gelungen, ein praxisgerechtes Standardwerk vorzulegen, das dem dermatologisch Interessierten, vom Arzt im Praktikum bis zum Facharzt, eine erstklassige Informationsquelle bei allen Fragen der Therapie bietet. Wünschenswert wäre es, wenn es den Herausgebern gelänge, in Zukunft einen weiteren Band zu veröffentlichen, der eine Übersicht über die übrigen therapeutischen Verfahren in der Dermatologie, wie beispielsweise Lichttherapie, Lasertherapie oder Strahlentherapie, bietet.

Dr. A. Niedermeier

\section{Korting HC, Sterry W, Hrsg. \\ Therapeutische Verfahren in der \\ Dermatologie - Dermatika und \\ Kosmetika}

Berlin - Wien: Blackwell-Wissenschafts-

Verlag, 2001. 784 Seiten, 298 DM

ISBN 3-89412-342-7 lich die so genannte Altershaut. Neben der Beschreibung der medikamentösen Therapie und Prophylaxe geben die Autoren tabellenförmig Hinweise zu den rückfettenden, juckreizstillenden oder keratolytischen Externa. Die sehr übersichtlichen Tabellen mit den zu empfehlenden Handelspräparaten zur Anwendung an Körper, Gesicht oder auf der Kopfhaut sind außerordentlich praktikabel und verdienen es, insbesondere für den ambulant tätigen Arzt zur Verfügung zu stehen, um sachkundig das schwierige Problem der richtigen Körperpflege mit dem Patienten zu besprechen.

$$
\text { Prof. Dr. K.-Ch. Bergmann }
$$

Schürer N, Kresken J

Die trockene Haut

Stuttgart: Wissenschaftliche Verlagsgesellschaft, 2000. 160 Seiten, 38 DM ISBN 3-8047-1776-4 